\title{
Progress in Research of Asymptomatic Primary Mitral Regurgitation Examination Methods: A Review of Literature
}

\author{
Chen Ma, ${ }^{1}$ Xie Fei, ${ }^{1}$ Weihao Ding, ${ }^{1}$ Zongwei Xiao ${ }^{2}$ \\ ${ }^{1}$ Graduate School of Zunyi Medical University, Zunyi, Guizhou, China; ${ }^{2}$ Department of Cardio-Thoracic Surgery, Chengdu Second \\ People's Hospital, Zunyi Medical University, Chengdu, Sichuan, China
}

\section{ABSTRACT}

Mitral regurgitation (MR) is a common valvular heart disease, which can be classified into primary and secondary, according to the cause. Primary mitral regurgitation (PMR) is caused by rheumatic fever, degenerative changes, valve prolapse, etc. The appearance of clinical symptoms has always been the best indicator of surgical intervention in patients with severe PMR, but for asymptomatic patients, the best treatment has been controversial. The choice of follow-up observation or early surgery has different results in different randomized studies. Two-dimensional echocardiography is the most commonly used detection method for evaluating $M R$, but its evaluation of the degree of reflux may be inaccurate, and there are differences in the outcomes of patients with asymptomatic PMR. Recent studies have shown that three-dimensional echocardiography, cardiac magnetic resonance, speckle-tracking echocardiography, brain natriuretic peptide, and exercise stress test can optimize the timing of surgery for asymptomatic patients and judge the asymptomatic of PMR.

\section{INTRODUCTION}

Due to the existence of cardiac compensation mechanism, cardiac function can maintain balance for a long time in patients with primary mitral regurgitation (PMR). However, long-term excessive volume load causes myocardial remodeling, which leads to left ventricular dysfunction and dilation, and eventually leads to pulmonary congestion-related clinical symptoms.

According to the clinical guidelines recommended by the European Society of Cardiology and the European Association of Cardiothoracic Surgery, the type I intervention indication for asymptomatic patients with severe PMR is left ventricular dysfunction [Baumgartner 2017], which is defined as left ventricular ejection fraction (LVEF) $\leq 60 \%$ and/or left ventricular end systolic diameter (LVDD), LVESD) $\geq$ $45 \mathrm{~mm}$. For patients with normal left ventricular function,

Received November 24, 2020; accepted December 14, 2020.

Correspondence: Zong Wei Xiao, Department of Cardio-Thoracic Surgery, Chengdu Second People's Hospital, Zunyi Medical University, Chengdu, Sichuan 600017, China (e-mail: zongweixiao@163.com). existing studies found that new onset of atrial fibrillation, resting pulmonary artery systolic pressure $>50 \mathrm{mmhg}$, flail valve leaflet, and left atrial volume index $\geq 60 \mathrm{ml} / \mathrm{m}^{2}$ during sinus rhythm are adverse prognoses after mitral valve surgery and risk factors affecting medium and long-term survival [Badhwar 2012; Ghoreishi 2011]. At present, the above indications have been included in the guidelines for class IIa surgery. Asymptomatic patients during follow up often are asymptomatic and risk-free in the surgical management of valve replacement. It will delay the patient's condition, increase the incidence of adverse cardiovascular events after surgery, and affect the medium and long-term survival of patients, especially in patients without systematic assessment and poor compliance; so how to identify high-risk patients early and carry out surgical intervention is very important for such patients.

\section{METHODS AND DISCUSSION}

A review of the literature concerning primary mitral regurgitation examination methods was performed.

Evaluation of primary mitral regurgitation by twodimensional echocardiography: Two-dimensional echocardiography (2DE) is an important technique for the diagnosis and evaluation of the severity and prognosis of valvular heart disease. It is closely related to the formulation of a clinical treatment plan. The degree of mitral regurgitation is affected by many factors, such as hemodynamic changes, cardiac cycle, left ventricular outflow tract obstruction, and arrhythmia and so on. Therefore, the degree of mitral regurgitation needs multi-section and multi-parameter synthesis, including the observation of valve shape, the range and size of color blood flow regurgitation beam, jet constriction orifice width, and the blood flow of superior vena cava. The quantitative evaluation relies on the proximal isovelocity surface area (PISA). However, PISA method has limitations. Studies have shown that the repeatability of PISA method in distinguishing severe and non-severe PMR is less than 50\% [Biner 2010], and the accuracy of PISA method in patients with non-circular regurgitation orifice, complex eccentric blood flow, multiple regurgitant beams and other problems is low, and it underestimates the mitral orifice regurgitation [Heo 2017]. These measurement differences affect the clinician's judgment on the timing of surgery to a certain extent.

The increase in LVESD and the decrease in LVEF are important risk factors for left ventricular insufficiency 
and death after surgery in PMR patients; however, studies have shown that there is a spherical mid-top left ventricular remodeling in severe PMR patients, which is manifested as contraction. As the terminal volume increases, the LVESD measurement value remains normal [Schiros 2012]. Therefore, a simple measurement of the LVESD change may not reflect the severity of the asymptomatic patient's condition, causing a misjudgment of the timing of surgery. Due to the increase in preload and blood flow to the left atrium during systole, the LVEF measured by 2DE is higher than the actual left ventricular systolic function, and there are considerable inter-observer differences [Thavendiranathan 2013], then LVEF $\leq 60 \%$ used as a fixed value for surgical intervention in asymptomatic patients may misjudge the timing of surgery; and the application of the following evaluation methods will reduce the occurrence of such misjudgments.

Assessment of asymptomatic patients with primary mitral regurgitation, three-dimensional echocardiography: Three-dimensional echocardiography (3DE) can directly display the three-dimensional shape of the heart cavity without being affected by the irregular geometric shape of the diseased heart cavity and overcomes the difference caused by the geometric assumptions of the heart cavity during the 2DE measurement. A three-dimensional model of the mitral valve is established by $3 \mathrm{DE}$ imaging, which can be used to evaluate the mitral valve device and analyze the causes of PMR, such as showing the diffuse thickening of the anterior and posterior leaflets caused by mucinous degeneration, or it shows the flail valve leaflet caused by the lack of local elastic fiber tissue, and the color Doppler blood flow signal profile of the regurgitant beam can be used to directly calculate the effective regurgitant flow of the mitral valve orifice [Chandra 2011]; and the transesophageal $3 \mathrm{DE}$ can be used. The unique perspective view can make up for the shortcomings of transthoracic $3 \mathrm{DE}$ and can also replace it when transthoracic images cannot be obtained. Because transesophageal 3DE improves the display of the mitral valve anatomy, it can be used in mitral and artificial mitral valves. The evaluation of pathological changes has unique advantages [Chandra 2011; Lang 2012]. Studies have shown that transesophageal $3 \mathrm{DE}$ is equivalent to cardiac multi-slice spiral computed tomography in accuracy and clinical feasibility in measuring mitral valve geometry [Shanks 2010]. In the quantitative assessment of PMR patients, 3DE uses PISA method to measure the accuracy of regurgitant flow and effective regurgitant orifice area is significantly better than 2DE and has good agreement among different observers [Heo 2017; de Agustín 2012]. Yingchoncharoen et al [Yingchoncharoen 2014] and other studies found that for asymptomatic or mild symptoms of severe PMR patients, left ventricular end systolic diameter volume index $\geq 40 \mathrm{ml} /$ $\mathrm{m}^{2}$ measured by $3 \mathrm{DE}$ is an independent indicator to predict left ventricular dysfunction and atrial fibrillation after mitral valve surgery, and left ventricular end systolic inner diameter volume index can be used to determine whether such patients have surgical indications. Although $3 \mathrm{DE}$ has many advantages in the evaluation of PMR patients, there is a lack of largescale randomized controlled trials to verify the practicability of the above-mentioned measurement indicators, and there is a lack of internationally unified relevant threshold indicators. The small volume and low time resolution of 3DE Doppler imaging also limit its use.

Assessment of asymptomatic patients with primary mitral regurgitation, cardiac magnetic resonance imaging: Cardiac magnetic resonance (CMR) can be used to evaluate cardiac function, cardiac volume, valve lesion, measure myocardial activity, evaluate coronary artery disease, and cardiac stress test [Kramer 2013]. In MR patients, CMR has the advantages of identifying MR mechanism, quantifying the severity of $\mathrm{MR}$, and determining its impact on cardiac remodeling. For patients with poor quality of $2 \mathrm{DE}$ examination or conflicting results with clinical results, CMR evaluation has the advantages of higher repeatability and myocardial viability, and these parameters are of great significance for surgical intervention [Zoghbi 2017; Cawley 2009]. A prospective multicenter study showed that CMR can more accurately assess the severity of PMR than 2DE, and the severity of reflux assessed by CMR has a good correlation with postoperative left ventricular remodeling $(\mathrm{R}=0.85)$. For asymptomatic patients who need to make surgical decisions, the results of CMR are very important [Uretsky 2015]; the degree of PMR is quantitatively analyzed by CMR. It was found that CMR can early identify patients who are going to have corresponding clinical symptoms or disease progression and need surgical intervention, and it is better than the reflux classification provided by $2 \mathrm{DE}$. Low reflux $\leq 55 \mathrm{ml}$ and reflux fraction $\leq 40 \%$ have high negative predictive value [Myerson 2016]; similarly, similar clinical results were observed in the study of Penicka et al [Penicka 2018], but their mitral regurgitation threshold determined by CMR was $50 \mathrm{ml}$, which may be related to the inclusion of some patients with moderate PMR in both studies. There are differences in the use of these parameters to evaluate the adverse outcomes and surgical indications of severe PMR. In addition, randomized controlled trials are needed to confirm these results to determine the clinical application value. Although the above studies have shown that CMR is superior to $2 \mathrm{DE}$ in evaluating PMR, CMR should be carried out without aortic regurgitation and other endovascular regurgitation and is greatly affected by irregular heart rhythm, which limits its use.

Assessment of asymptomatic patients with primary mitral regurgitation, speckle tracking echocardiography: Speckle tracking echocardiography is a new technique to evaluate the local and global function of the heart. Its principle is to reflect the systolic and diastolic function of the heart by tracking the "spot" changes of the central muscle in the cardiac cycle. It has the advantage of evaluating the cardiac function from the ultrastructural changes of different layers of myocardium strip. At this stage, it is believed that Global Longitudinal Strain (GLS) is a conventional indicator for evaluating the overall function of the left ventricle. The LV-GLS is an early and sensitive detection for left ventricular insufficiency [Nagueh 2016]; some studies have shown that in patients with asymptomatic severe PMR (LVEF > 60\%), the reduction of myocardial systolic velocity, LV-GLS and strain rate is used to identify subclinical left ventricular dysfunction [Florescu 2012]. In the study of Magne et al [Magne 2014], 
and some other study, asymptomatic PMR patients with an increase in left ventricular GLS $\geq 2 \%$ before and after exercise stress test were considered to have left ventricular systolic reserve function. During the follow up, the probability of adverse cardiac events (cardiac death, mitral valve surgery, heart failure requiring hospitalization, and acute pulmonary edema) was significantly increased ( $69 \pm 7 \%$ versus $42 \pm 8 \%$ ) in non PMR patients. As an independent risk factor for cardiac events (HR $=2.27,95 \%$ CI: $1.05-4.76, P=0.037)$, left ventricular systolic reserve can improve risk stratification and clinical decision-making in these patients.

The evaluation of left atrial function currently uses left atrial longitudinal strain (LAS) and strain rate. Related parameters include left atrial systolic longitudinal peak strain (LASct), global diastolic left atrial peak strain (LAScd), and left atrium maximum longitudinal peak strain (LASr), and the corresponding strain rate [Badano 2018]; in patients with severe PMR indications for surgery, the median of LASct, LASdt, and LASr were significantly reduced. Regression analysis showed that LASr is an independent risk factor for identifying surgical indications [Debonnaire 2013]. In patients with asymptomatic PMR, LASr is closely related to the degree of atrial fibrosis and atrial remodeling [Cameli 2013]. The reduction of LASr and the peak left atrial strain rate, during the whole diastolic period, suggests that cardiogenic death and mitral valve surgery and hospitalization due to heart failure are related to the observation endpoint [Yang 2015] and can be used as an independent tool to determine the best timing of surgery for such patients.

Although speckle tracking echocardiography has an independent predictive effect on asymptomatic patients with severe PMR and strengthens the understanding of the physiology and overall deformation of the myocardium, it is greatly affected by instruments and software, and the measurement parameters lack a unified international consensus. Its application in clinical and scientific research needs further research and promotion.

Assessment of asymptomatic patients with primary mitral regurgitation, brain natriuretic peptide: Brain natriuretic peptide (BNP) is a member of the natriuretic peptide family. It is secreted by the left ventricle, when the ventricular wall tension is increased. It plays a role in natriuretic diuresis, vasodilation, reducing the load before and after loading, and improving myocardial remodeling. Studies have shown that BNP can be used to identify early left ventricular dysfunction and increase with the severity of MR [Abdel 2016]. In the follow up of asymptomatic severe PMR patients, compared with the non-event patients, BNP can be used to identify early left ventricular dysfunction. The median level of BNP was significantly increased in patients with clinical symptoms, left ventricular dysfunction, atrial fibrillation, and pulmonary artery systolic pressure $>50 \mathrm{mmHg}$. BNP $(<79 \mathrm{pg} / \mathrm{ml})$ had a higher negative predictive value (98\%) [Klaar 2011], while similar results were observed in the prospective study of Pizarro et al [Pizarro 2009]. In the deduced cohort $(\mathrm{N}=$ 167), asymptomatic severe PMR patients with $\mathrm{BNP} \geq 105 \mathrm{pg} /$ $\mathrm{ml}$ had a higher risk of cardiovascular events (congestive heart failure, left ventricular dysfunction, and death) within
48 months $(76 \%)$. In the subsequent validation cohort $(\mathrm{N}=$ $102)$, the risk ratio of cardiovascular events within 48 months was $66 \%$ versus $4 \%$, and the sensitivity, specificity, negative and positive predictive values of $\mathrm{BNP} \geq 105 \mathrm{pg} / \mathrm{ml}$ were $80 \%$, $93 \%, 94 \%, 76 \%$ and $85 \%, 89 \%, 96 \%$ and $66 \%$, respectively. However, Magne et al [Magne 2012] thought that the BNP change level before and after exercise may have a more predictive effect than the resting BNP level, such patients with BNP change value $>64 \mathrm{pg} / \mathrm{ml}$ before and after exercise have higher risk of cardiovascular events (death, heart failure, mitral valve surgery for symptoms, left ventricular dilatation, or dysfunction), but there is no validation group to verify, and this study included more patients with moderate PMR were in the $\mathrm{BNP}<64 \mathrm{pg} / \mathrm{ml}$ group, which may underestimate the predictive effect of BNP on the development of severe PMR. However, it is undeniable that both resting BNP and BNP before and after exercise can independently predict the outcome of such patients. The continuous increase of BNP level (even in the normal range) is a sign of left ventricular dysfunction, reflecting the increased dependence on cardiac reserve function. However, there are still too many small sample sizes, lack of consistency of observation end points, and no unity prediction model and other issues need comprehensive research to confirm.

Another index: Precursor of BNP (NT proBNP) is a kind of straight chain protein produced simultaneously with BNP. Compared with BNP, NT proBNP lacks biological activity, but has the advantage of longer half-life. Due to the same molar secretion with BNP, NT proBNP has similar predictive effect with BNP.

Assessment of asymptomatic patients with primary mitral regurgitation, plasma protein: Recently, the role of protein in disease prediction has been emphasized. By comparing the expression of thousands of proteins in PMR patients and normal people, the proteome related to the prognosis of PMR was obtained. High density lipoprotein, apolipoprotein $\mathrm{A} 1$, and haptoglobin - $\alpha 2$ were considered to be in direct proportion to the severity of reflux, and apolipoprotein A1 was an independent predictor of severe PMR [Deroyer 2015]. In the study of Tan et al [Tan 2013], the levels of plasma haptoglobin, platelet basic protein and complement C4B in asymptomatic PMR patients were lower than those in the normal control group, but some patients with moderate PMR were included in this study, and the clinical adverse outcomes were not observed and analyzed. In these studies, it can be confirmed that due to the damage of mitral valve or subvalvular device, the intracardiac blood flow can be confirmed, monitoring the changes of plasma proteome concentration can predict the progress of the disease, but more research is needed to confirm.

Assessment of asymptomatic patients with primary mitral regurgitation, exercise stress test: The progression of PMR is a complex dynamic process, and exercise stress test can be used for the evaluation of asymptomatic or nonspecific symptoms, especially for those patients with normal left ventricular function under resting state, the combination of exercise load test, and clinical monitoring indicators can help to evaluate the progress of the disease [Henri 2014]; in PMR, exercise 
echocardiography is abnormal (exercise metabolic equivalent $<5$, blood pressure rise $<20 \mathrm{mmhg}$, exercise pulmonary artery pressure $>60 \mathrm{mmhg}$ ) suggested that the risk of death or hospitalization due to heart failure within two years was increased, and it can be used as a part of routine follow up for asymptomatic PMR patients [Sharma 2015]. Among asymptomatic patients with severe PMR (LVEF > 60\%), Magne et al [Magne 2014; Magne 2012] considered that patients with GLS increase $\geq 2 \%$ before and after exercise load test have better left ventricular systolic reserve function, the probability of disease deterioration is lower, and the change value of $\mathrm{BNP}<64 \mathrm{pg} / \mathrm{ml}$ has lower risk of cardiovascular events.

\section{CONCLUSION}

Due to the existence of compensation mechanism, PMR patients have been asymptomatic for many years, but eventually there will be adverse clinical results such as left atrial enlargement, left ventricular dilatation, left ventricular dysfunction, atrial fibrillation, pulmonary hypertension, and other adverse clinical results. With the continuous development of medical technology and the progress and maturity of detection methods, clinicians can accurately predict asymptomatic patients with PMR through new evaluation methods and indicators for disease progression, refine risk stratification, and develop more reasonable treatment strategy.

\section{REFERENCES}

Abdel Fattah EM, Girgis HY, El Khashab K, et al. 2016. B-type Natriuretic Peptide as an Index of Symptoms and Severity of Chronic Rheumatic Mitral Regurgitation[J]. Heart Views. 17(1):7-12.

Badano LP, Kolias TJ, Muraru D, et al. 2018. Standardization of left atrial, right ventricular, and right atrial deformation imaging using twodimensional speckle tracking echocardiography: a consensus document of the EACVI/ASE/Industry Task Force to standardize deformation imaging[J]. Eur Heart J Cardiovasc Imaging. 19(6):591-600.

Badhwar V, Peterson ED, Jacobs JP, et al. 2012. Longitudinal outcome of isolated mitral repair in older patients: results from 14,604 procedures performed from 1991 to 2007[J]. Ann Thorac Surg. 94(6):1870-7; discussion 1877-9.

Baumgartner H, Falk V, Bax JJ, et al. 2017. 2017 ESC/EACTS Guidelines for the management of valvular heart disease[J]. Eur Heart J. 38(36):2739-2791.

Biner S, Rafique A, Rafii F, et al. 2010. Reproducibility of proximal isovelocity surface area, vena contracta, and regurgitant jet area for assessment of mitral regurgitation severity[J]. JACC Cardiovasc Imaging. 3(3):235-43.

Cameli M, Lisi M, Righini FM, et al. 2013. Usefulness of atrial deformation analysis to predict left atrial fibrosis and endocardial thickness in patients undergoing mitral valve operations for severe mitral regurgitation secondary to mitral valve prolapse[J]. Am J Cardiol. 111(4):595-601.

Cawley PJ, Maki JH, Otto CM. 2009. Cardiovascular magnetic resonance imaging for valvular heart disease: technique and validation[J]. Circulation. 119(3):468-78.
Chandra S, Salgo IS, Sugeng L, et al. 2011. A three-dimensional insight into the complexity of flow convergence in mitral regurgitation: adjunctive benefit of anatomic regurgitant orifice area[J]. Am J Physiol Heart Circ Physiol. 301(3):H1015-24.

de Agustín JA, Marcos-Alberca P, Fernandez-Golfin C, et al. 2012. Direct measurement of proximal isovelocity surface area by single-beat three-dimensional color Doppler echocardiography in mitral regurgitation: a validation study[J]. J Am Soc Echocardiogr. 25(8):815-23.

Debonnaire P, Leong DP, Witkowski TG, et al. 2013. Left atrial function by two-dimensional speckle-tracking echocardiography in patients with severe organic mitral regurgitation: association with guidelines-based surgical indication and postoperative (long-term) survival[J]. J Am Soc Echocardiogr. 26(9):1053-62.

Deroyer C, Magne J, Moonen M, et al. 2015. New biomarkers for primary mitral regurgitation[J]. Clin Proteomics. 12:25.

Florescu M, Benea DC, Rimbas RC, et al. 2012. Myocardial systolic velocities and deformation assessed by speckle tracking for early detection of left ventricular dysfunction in asymptomatic patients with severe primary mitral regurgitation[J]. Echocardiography. 29(3):326-33.

Ghoreishi M, Evans CF, DeFilippi CR, et al. 2011. Pulmonary hypertension adversely affects short- and long-term survival after mitral valve operation for mitral regurgitation: implications for timing of surgery[J]. J Thorac Cardiovasc Surg. 142(6):1439-52.

Henri C, Piérard LA, Lancellotti P, et al. 2014. Exercise testing and stress imaging in valvular heart disease[J]. Can J Cardiol. 30(9):1012-26.

Heo R, Son JW, Ó Hartaigh B, et al. 2017. Clinical Implications of Three-Dimensional Real-Time Color Doppler Transthoracic Echocardiography in Quantifying Mitral Regurgitation: A Comparison with Conventional Two-Dimensional Methods[J]. J Am Soc Echocardiogr. 30(4):393-403.e7.

Klaar U, Gabriel H, Bergler-Klein J, et al. 2011. Prognostic value of serial B-type natriuretic peptide measurement in asymptomatic organic mitral regurgitation[J]. Eur J Heart Fail. 13(2):163-9.

Kramer CM, Barkhausen J, Flamm SD, et al. 2013. Standardized cardiovascular magnetic resonance (CMR) protocols 2013 update[J]. J Cardiovasc Magn Reson. 15:91.

Lang RM, Badano LP, Tsang W, et al. 2012. EAE/ASE recommendations for image acquisition and display using three-dimensional echocardiography[J]. Eur Heart J Cardiovasc Imaging. 13(1):1-46.

Magne J, Mahjoub H, Dulgheru R, et al. 2014. Left ventricular contractile reserve in asymptomatic primary mitral regurgitation[J]. Eur Heart J. 35(24):1608-16.

Magne J, Mahjoub H, Pibarot P, et al. 2012. Prognostic importance of exercise brain natriuretic peptide in asymptomatic degenerative mitral regurgitation[J]. Eur J Heart Fail. 14(11):1293-302.

Myerson SG, d'Arcy J, Christiansen JP, et al. 2016. Determination of Clinical Outcome in Mitral Regurgitation With Cardiovascular Magnetic Resonance Quantification[J]. Circulation. 133(23):2287-96.

Nagueh SF, Smiseth OA, Appleton CP, et al. 2016. Recommendations for the Evaluation of Left Ventricular Diastolic Function by Echocardiography: An Update from the American Society of Echocardiography and the European Association of Cardiovascular Imaging[J]. Eur Heart J Cardiovasc Imaging. 17(12):1321-1360.

Penicka M, Vecera J, Mirica DC, et al. 2018. Prognostic Implications of Magnetic Resonance-Derived Quantification in Asymptomatic Patients With Organic Mitral Regurgitation: Comparison With 
Doppler Echocardiography-Derived Integrative Approach[J]. Circulation. 137(13):1349-1360.

Pizarro R, Bazzino OO, Oberti PF, et al. 2009. Prospective validation of the prognostic usefulness of brain natriuretic peptide in asymptomatic patients with chronic severe mitral regurgitation[J]. J Am Coll Cardiol. 54(12):1099-106.

Schiros CG, Dell'Italia LJ, Gladden JD, et al. 2012. Magnetic resonance imaging with 3-dimensional analysis of left ventricular remodeling in isolated mitral regurgitation: implications beyond dimensions[J]. Circulation. 125(19):2334-42.

Shanks M, Delgado V, Ng AC, et al. 2010. Mitral valve morphology assessment: three-dimensional transesophageal echocardiography versus computed tomography[J]. Ann Thorac Surg. 90(6):1922-9.

Sharma V, Newby DE, Stewart RA, et al. 2015. Exercise stress echocardiography in patients with valvular heart disease[J]. Echo Res Pract. 2(3):89-98.

Tan HT, Ling LH, Dolor-Torres MC, et al. 2013. Proteomics discovery of biomarkers for mitral regurgitation caused by mitral valve prolapse[J]. J Proteomics. 94:337-45.
Thavendiranathan P, Popovi ZB, Flamm SD, et al. 2013. Improved interobserver variability and accuracy of echocardiographic visual left ventricular ejection fraction assessment through a self-directed learning program using cardiac magnetic resonance images[J]. J Am Soc Echocardiogr. 26(11):1267-73.

Uretsky S, Gillam L, Lang R, et al. 2015. Discordance between echocardiography and MRI in the assessment of mitral regurgitation severity: a prospective multicenter trial[J]. J Am Coll Cardiol. 65(11):1078-88.

Yang LT, Liu YW, Shih JY, et al. 2015. Predictive value of left atrial deformation on prognosis in severe primary mitral regurgitation[J]. J Am Soc Echocardiogr. 28(11):1309-1317.e4.

Yingchoncharoen T, Negishi T, Stanton T, et al. 2014. Incremental value of three-dimensional echocardiography in the evaluation of left ventricular size in mitral regurgitation: a follow-up study after mitral valve surgery[J]. J Am Soc Echocardiogr. 27(6):608-15.

Zoghbi WA, Adams D, Bonow RO, et al. 2017. Recommendations for Noninvasive Evaluation of Native Valvular Regurgitation: A Report from the American Society of Echocardiography Developed in Collaboration with the Society for Cardiovascular Magnetic Resonance[J]. J Am Soc Echocardiogr. 30(4):303-371. 